\title{
Magnetic impurity inspired Abelian Higgs vortices
}

\author{
Xiaosen $\mathbf{H a n}^{a, b}$ and Yisong Yang ${ }^{c, d, 1}$ \\ ${ }^{a}$ Institute of Contemporary Mathematics, School of Mathematics, Henan University, \\ Kaifeng, Henan 475004, P.R. China \\ ${ }^{b}$ Dipartimento di Matematica, Università di Roma "Tor Vergata", \\ Via della Ricerca Scientifica, 00133 Rome, Italy ${ }^{2}$ \\ ${ }^{c}$ Department of Mathematics, Tandon School, New York University, \\ Brooklyn, New York 11201, U.S.A. \\ ${ }^{d}$ NYU-ECNU Institute of Mathematical Sciences, New York University — Shanghai, \\ 3663 North Zhongshan Road, Shanghai 200062, P.R. China \\ E-mail: hanxiaosen@henu.edu.cn, yisongyang@nyu.edu
}

ABSTRACT: Inspired by magnetic impurity considerations some broad classes of Abelian Higgs and Chern-Simons-Higgs BPS vortex equations are derived and analyzed.

Keywords: Field Theories in Lower Dimensions, Solitons, Monopoles, and Instantons, Chern-Simons Theories

ArXiv EPRINT: 1510.07077

\footnotetext{
${ }^{1}$ Corresponding author.

${ }^{2}$ Present address.
} 


\section{Contents}

1 Introduction 1

2 Abelian Higgs vortices and impurities $\quad 2$

3 Generalized Abelian Higgs vortices 4

4 Chern-Simons vortices in presence of magnetic impurities 6

$\begin{array}{lll}5 & \text { Generalized Chern-Simons-Higgs vortices } & 7\end{array}$

6 Solutions of vortex equations $\quad 9$

6.1 Vortex equations in presence of $\sigma(x)$-source terms 10

$\begin{array}{lll}6.2 & \text { Abelian Higgs vortex equations } & 12\end{array}$

$\begin{array}{lll}6.3 & \text { Chern-Simons vortex equations } & 14\end{array}$

$\begin{array}{lll}7 & \text { Conclusions } & 15\end{array}$

\section{Introduction}

The Nielsen-Olesen vortices [29] are of important relevance in theoretical physics. In superconductivity theory, they appear as spatially periodic topological defects, known as the Abrikosov vortices [1], allowing partial penetration of magnetic field, which characterizes the onset of type-II superconductivity. Such vortices, when viewed in a three-dimensional formalism, give rise to string-like structure for the concentration of magnetic field, often referred to as the Nielsen-Olesen strings. These magnetic strings mediate the interaction between a monopole and an anti-monopole, hypothetically immersed in a type-II superconductor, so that the attractive force between the pair remains constant and the energy required to separate the pair is proportional to the distance between the two magnetic poles. As a result, it would require infinite energy to break free a bonded pair of a monopole and an anti-monopole placed in a type-II superconductor. This phenomenon, known as the monopole confinement, was proposed by Mandelstam [23, 24], Nambu [27], and 't Hooft [40, 41], and has served as a profound source of thoughts [33] for the understanding of quark confinement where the underlying Abelian Higgs model platform is extended into various supersymmetric gauge field theory models and the classical Meissner effect is elevated into its supersymmetric versions so that the Nielsen-Olesen magnetic strings become colored ones $[3,7,8,10,12,14,15,26,35,36]$. See $[9,11,21,34,37,38,44,45]$ for surveys and further literature.

The full vortex equations, even in the simplest Abelian Higgs model, are too complicated to analyze to obtain a complete interaction picture. Fortunately, one may often 
extract sufficient insight [38, 44, 45] from exploring a so-called BPS structure after the works of Bogomol'nyi [4] and Prasad and Sommerfeld [32].

In a recent study [17], electric and magnetic impurities are considered in the Abelian Higgs model in the context of supersymmetric field theories. It is shown that the usual BPS structure is preserved in the presence of such impurities. In [46], it is demonstrated that magnetic impurities may be viewed as heavy, frozen vortices sitting in an additional Abelian gauge group, so that the interaction of the Abelian Higgs vortices with impurities may be described in the framework of a product Abelian gauge field theory containing two scalar fields $q$ and $p$ with doublet charges $(+1,-1)$ and $(0,+1)$, respectively.

In this paper we first show that the model of Tong and Wong [46] belongs to a general product Abelian Higgs model with two scalar fields carrying charges $(a, b)$ and $(c, d)$ for arbitrary $a, b, c, d \in \mathbb{R}$. We then show that this generalization may be made to the wellknown Abelian Chern-Simons-Higgs theory initially developed by Hong, Kim, and Pac [16], and Jackiw and Weinberg [18]. In this latter context we begin by extending the work [16, 18] to include a magnetic $\sigma$-source term in the spirit of $[17,46]$. We next expand our extension of the product Abelian Higgs model of Tong-Wong [46] to derive a product Abelian ChernSimons-Higgs model, under the non-degeneracy condition $a d-b c \neq 0$. Our main emphasis is to maintain the BPS structures in all these developments.

The contents of the rest of the paper is outlined as follows. In the next section, we recall some of the results in the study [46] which inspire our present work and introduce our main notation. In section 3, we develop our extended product Abelian gauge theory. In section 4, we consider the Abelian Chern-Simons-Higgs model in the presence of a magnetic source term. In section 5, we develop a product Abelian Chern-Simons-Higgs gauge theory. In section 6, we present a series of existence, sometimes including uniqueness as well, results for the extended BPS vortex equations obtained. In section 7 , we draw conclusions.

\section{Abelian Higgs vortices and impurities}

In normalized units, the Abelian Higgs model defined over the standard Minkowski spacetime $\mathbb{R}^{2,1}$ of signature (+ - -), of a Bogomol'nyi-Prasad-Sommerfield (BPS) structure [4, 32], is given by the Lagrangian density

$$
\mathcal{L}=-\frac{1}{4 \kappa} F_{\mu \nu} F^{\mu \nu}+D_{\mu} \phi \overline{D^{\mu} \phi}-\frac{\kappa}{2}\left(|\phi|^{2}-\zeta\right)^{2}
$$

where the connection or gauge-covariant derivative $D_{\mu}$ reads

$$
D_{\mu} \phi=\partial_{\mu} \phi-\mathrm{i} A_{\mu} \phi
$$

$\phi$ is a complex-valued Higgs scalar field carrying charge $+1, F_{\mu \nu}=\partial_{\mu} A_{\nu}-\partial_{\nu} A_{\mu}$ the electromagnetic field induced from the real-valued gauge field $A_{\mu}(\mu=0,1,2)$, and $\kappa, \zeta>0$ are coupling constants such that $\sqrt{\kappa \zeta}$ gives the mass of the Higgs particle and $\sqrt{\zeta}$ measures the energy scale of the spontaneously broken symmetry. The equations of motion of (2.1) are

$$
\begin{aligned}
D_{\mu} D^{\mu} \phi & =\kappa\left(|\phi|^{2}-\zeta\right) \phi, \\
\frac{1}{\kappa} \partial^{\nu} F_{\mu \nu} & =\mathrm{i}\left(\bar{\phi} D_{\mu} \phi-\phi \overline{D_{\mu} \phi}\right),
\end{aligned}
$$


which are hard to solve. In the static limit, all finite-energy solutions of these equations must be electrically neutral, $A_{0}=0$, a statement known as the Julia-Zee theorem [20,39], such that the equations can be replaced by the BPS system

$$
\begin{aligned}
F_{12} \pm \kappa\left(|\phi|^{2}-\zeta\right) & =0, \\
D_{1} \phi \pm \mathrm{i} D_{2} \phi & =0 .
\end{aligned}
$$

In $[17,46]$, the model $(2.1)$ is extended to include a static magnetic source term $\sigma(x)$ $\left(x=\left(x^{1}, x^{2}\right)\right)$ so that the static energy density without electricity $\left(A_{0}=0\right)$ assumes the form

$$
\mathcal{E}=\frac{1}{2 \kappa} F_{12}^{2}+\left|D_{j} \phi\right|^{2}+\frac{\kappa}{2}\left(|\phi|^{2}-\zeta-\sigma(x)\right)^{2} \mp \sigma(x) F_{12},
$$

whose equations of motion are

$$
\begin{aligned}
D_{j} D_{j} \phi & =\kappa\left(|\phi|^{2}-\zeta-\sigma(x)\right) \phi, \\
\partial_{j} F_{12} & =\kappa \varepsilon^{j k} \mathrm{i}\left(\bar{\phi} D_{k} \phi-\phi \overline{D_{k} \phi}\right) \pm \kappa \partial_{j} \sigma .
\end{aligned}
$$

On the other hand, by the BPS quadrature procedure, we have

$$
\begin{aligned}
E & =\int_{\mathbb{R}^{2}} \mathcal{E} \mathrm{d} x \\
& =\int_{\mathbb{R}^{2}}\left\{\frac{1}{2 \kappa}\left(F_{12} \pm \kappa\left(|\phi|^{2}-\zeta-\sigma\right)\right)^{2}+\left|D_{1} \phi \pm \mathrm{i} D_{2} \phi\right|^{2}\right\} \mathrm{d} x \pm \zeta \int_{\mathbb{R}^{2}} F_{12} \mathrm{~d} x \\
& \geq 2 \pi \zeta|N|,
\end{aligned}
$$

where $N$ is the winding number of the Higgs field $\phi$ which also defines the quantized flux:

$$
\Phi=\int_{\mathbb{R}^{2}} F_{12} \mathrm{~d} x=2 \pi N
$$

Moreover, the energy lower bound given in $(2.10)$ is attained when $\left(\phi, A_{j}\right)$ solves the BPS equations

$$
\begin{aligned}
D_{1} \phi \pm \mathrm{i} D_{2} \phi & =0, \\
F_{12} \pm \kappa\left(|\phi|^{2}-\zeta-\sigma(x)\right) & =0,
\end{aligned}
$$

whose solutions carry the energy or vortex tension

$$
E=2 \pi \zeta|N|
$$

which is seen to be indifferent to the magnetic source term.

It may be examined directly that (2.12)-(2.13) imply (2.8)-(2.9).

In [46], Tong and Wong show how the magnetic impurities as prescribed in (2.7) can be regarded as heavy, frozen vortices residing in another Abelian gauge group. Such a formalism is a theory built over the product Abelian gauge group, $\hat{U}(1) \times \tilde{U}(1)$, and accommodates two charged scalar fields, $q$ and $p$, carrying charges $(+1,-1)$ and $(0,+1)$, respectively, so that the gauge-covariant derivatives are

$$
D_{\mu} q=\partial_{\mu} q-\mathrm{i} \hat{A}_{\mu} q+\mathrm{i} \tilde{A}_{\mu} q, \quad D_{\mu} p=\partial_{\mu} p-\mathrm{i} \tilde{A}_{\mu} p,
$$


and the Lagrangian density reads

$$
\begin{aligned}
\mathcal{L}= & -\frac{1}{4 \kappa} \hat{F}_{\mu \nu} \hat{F}^{\mu \nu}-\frac{1}{4 \tilde{\kappa}} \tilde{F}_{\mu \nu} \tilde{F}^{\mu \nu}+D_{\mu} q \overline{D^{\mu} q}+D_{\mu} p \overline{D^{\mu} p} \\
& +\frac{\kappa}{2}\left(|q|^{2}-\zeta\right)^{2}+\frac{\tilde{\kappa}}{2}\left(-|q|^{2}+|p|^{2}-\tilde{\zeta}\right)^{2}
\end{aligned}
$$

where $\hat{F}_{\mu \nu}=\partial_{\mu} \hat{A}_{\nu}-\partial_{\nu} \hat{A}_{\mu}$ and $\tilde{F}_{\mu \nu}=\partial_{\mu} \tilde{A}_{\nu}-\partial_{\nu} \tilde{A}_{\mu}$, and $\tilde{\kappa}>0$ and $\tilde{\zeta}>-\zeta$ are two additional coupling constants. The vacuum state of the theory is characterized by $|q|^{2}=\zeta$, $|p|^{2}=\zeta+\tilde{\zeta}$, and the static equations of motion in the temporal gauge enjoy the BPS reduction $[46]$ :

$$
\begin{aligned}
\hat{F}_{12} \pm \kappa\left(|q|^{2}-\zeta\right) & =0, \\
D_{1} q \pm \mathrm{i} D_{2} q & =0, \\
\tilde{F}_{12} \pm \tilde{\kappa}\left(-|q|^{2}+|p|^{2}-\tilde{\zeta}\right) & =0, \\
D_{1} p \pm \mathrm{i} D_{2} p & =0 .
\end{aligned}
$$

In the present work our main concern is to uncover some extended classes of the BPS equations in the same framework of Tong and Wong [46]. First we show that the above Tong-Wong BPS vortex equations belong to a broader formalism that accommodates general doublet charges of the form $(a, b),(c, d)$ for arbitrary real parameters $a, b, c, d$. Next we extend our study to the Abelian Chern-Simons model $[16,18]$. In this context we first derive the Chern-Simons BPS vortex equations in the presence of a magnetic impurity term in view of the studies $[17,46]$. We then obtain BPS vortex equations for the scalar fields carrying charges $(a, b)$ and $(c, d)$ as in the Abelian Higgs model case considered earlier, subject to the non-degeneracy condition

$$
a d-b c \neq 0 .
$$

\section{Generalized Abelian Higgs vortices}

Let $\hat{A}_{\mu}$ and $\tilde{A}_{\mu}$ be real-valued gauge fields lying in the Lie algebras of $\hat{\mathrm{U}}(1)$ and $\tilde{\mathrm{U}}(1)$, respectively, and $\hat{F}_{\mu \nu}=\partial_{\mu} \hat{A}_{\nu}-\partial_{\nu} \hat{A}_{\mu}$ and $\tilde{F}_{\mu \nu}=\partial_{\mu} \tilde{A}_{\nu}-\partial_{\nu} \tilde{A}_{\mu}$ the induced electromagnetic fields. The gauge-covariant derivatives on the scalar fields $q$ and $p$ carrying charges $(a, b)$ and $(c, d)$, respectively, are defined by

$$
D_{\mu} q=\partial_{\mu} q-\mathrm{i}\left(a \hat{A}_{\mu}+b \tilde{A}_{\mu}\right) q, \quad D_{\mu} p=\partial_{\mu} p-\mathrm{i}\left(c \hat{A}_{\mu}+d \tilde{A}_{\mu}\right) p, \quad \mu=0,1,2,
$$

where $a, b, c, d$ are real coupling parameters. Note that in this section we will not assume that these parameters satisfy (2.21) for full generality.

Motivated by [46], we consider the following Lagrangian density

$$
\mathcal{L}=-\frac{1}{4 \kappa} \hat{F}_{\mu \nu} \hat{F}^{\mu \nu}-\frac{1}{4 \lambda} \tilde{F}_{\mu \nu} \tilde{F}^{\mu \nu}+D_{\mu} q \overline{D^{\mu} q}+D_{\mu} p \overline{D^{\mu} p}-V\left(|q|^{2},|p|^{2}\right),
$$

where the potential density $V$ assumes the quartic form

$$
V\left(|q|^{2},|p|^{2}\right)=\frac{\kappa}{2}\left(a\left[|q|^{2}-\xi\right]+c\left[|p|^{2}-\zeta\right]\right)^{2}+\frac{\lambda}{2}\left(b\left[|q|^{2}-\xi\right]+d\left[|p|^{2}-\zeta\right]\right)^{2},
$$


with $\kappa, \lambda>0$ being constants. The Euler-Lagrange equations of (3.2) are

$$
\begin{aligned}
D_{\mu} D^{\mu} q & =a \kappa\left(a\left[|q|^{2}-\xi\right]+c\left[|p|^{2}-\zeta\right]\right) q+b \lambda\left(b\left[|q|^{2}-\xi\right]+d\left[|p|^{2}-\zeta\right]\right) q, \\
D_{\mu} D^{\mu} p & =c \kappa\left(a\left[|q|^{2}-\xi\right]+c\left[|p|^{2}-\zeta\right]\right) p+d \lambda\left(b\left[|q|^{2}-\xi\right]+d\left[|p|^{2}-\zeta\right]\right) p, \\
\frac{1}{\kappa} \partial^{\nu} \hat{F}_{\mu \nu} & =\mathrm{i}\left(a\left[\bar{q} D_{\mu} q-q \overline{D_{\mu} q}\right]+c\left[\bar{p} D_{\mu} p-p \overline{D_{\mu} p}\right]\right), \\
\frac{1}{\lambda} \partial^{\nu} \tilde{F}_{\mu \nu} & =\mathrm{i}\left(b\left[\bar{q} D_{\mu} q-q \overline{D_{\mu} q}\right]+d\left[\bar{p} D_{\mu} p-p \overline{D_{\mu} p}\right]\right),
\end{aligned}
$$

which are complicated.

On the other hand, note that we have the identities

$$
\begin{aligned}
& \left|D_{j} q\right|^{2}=\left|D_{1} q \pm \mathrm{i} D_{2} q\right|^{2} \pm \mathrm{i}\left(\partial_{1}\left[q \overline{D_{2} q}\right]-\partial_{2}\left[q \overline{D_{1} q}\right]\right) \pm\left(a \hat{F}_{12}+b \tilde{F}_{12}\right)|q|^{2}, \\
& \left|D_{j} p\right|^{2}=\left|D_{1} p \pm \mathrm{i} D_{2} p\right|^{2} \pm \mathrm{i}\left(\partial_{1}\left[p \overline{D_{2} p}\right]-\partial_{2}\left[p \overline{D_{1} p}\right]\right) \pm\left(c \hat{F}_{12}+d \tilde{F}_{12}\right)|p|^{2} .
\end{aligned}
$$

Thus, in view of (3.8) and (3.9), we see that in the electrically neutral situation, $\hat{A}_{0}=0$, $\tilde{A}_{0}=0$, the static energy density becomes

$$
\begin{aligned}
\mathcal{E}= & \frac{1}{2 \kappa} \hat{F}_{12}^{2}+\frac{1}{2 \lambda} \tilde{F}_{12}^{2}+\left|D_{j} q\right|^{2}+\left|D_{j} p\right|^{2}+V\left(|q|^{2},|q|^{2}\right) \\
= & \frac{1}{2 \kappa}\left(\hat{F}_{12} \pm \kappa\left(a\left[|q|^{2}-\xi\right]+c\left[|p|^{2}-\zeta\right]\right)\right)^{2}+\frac{1}{2 \lambda}\left(\tilde{F}_{12} \pm \lambda\left(b\left[|q|^{2}-\xi\right]+d\left[|p|^{2}-\zeta\right]\right)\right)^{2} \\
& \mp \hat{F}_{12}\left(a\left[|q|^{2}-\xi\right]+c\left[|p|^{2}-\zeta\right]\right) \mp \tilde{F}_{12}\left(b\left[|q|^{2}-\xi\right]+d\left[|p|^{2}-\zeta\right]\right) \\
& +\left|D_{1} q \pm \mathrm{i} D_{2} q\right|^{2}+\left|D_{1} p \pm \mathrm{i} D_{2} p\right|^{2} \pm \mathrm{i}\left(\partial_{1}\left[q \overline{D_{2} q}\right]-\partial_{2}\left[q \overline{D_{1} q}\right]\right) \pm\left(a \hat{F}_{12}+b \tilde{F}_{12}\right)|q|^{2} \\
& \pm \mathrm{i}\left(\partial_{1}\left[p \overline{D_{2} p}\right]-\partial_{2}\left[p \overline{D_{1} p}\right]\right) \pm\left(c \hat{F}_{12}+d \tilde{F}_{12}\right)|p|^{2} \\
= & \frac{1}{2 \kappa}\left(\hat{F}_{12} \pm \kappa\left(a\left[|q|^{2}-\xi\right]+c\left[|p|^{2}-\zeta\right]\right)\right)^{2}+\frac{1}{2 \lambda}\left(\tilde{F}_{12} \pm \lambda\left(b\left[|q|^{2}-\xi\right]+d\left[|p|^{2}-\zeta\right]\right)\right)^{2} \\
& +\left|D_{1} q \pm \mathrm{i} D_{2} q\right|^{2} \pm(a \xi+c \zeta) \hat{F}_{12} \pm(b \xi+d \zeta) \tilde{F}_{12} \\
& +\left|D_{1} p \pm \mathrm{i} D_{2} p\right|^{2} \pm \mathrm{i}\left(\partial_{1}\left[q \overline{D_{2} q}\right]-\partial_{2}\left[q \overline{D_{1} q}\right]\right) \pm \mathrm{i}\left(\partial_{1}\left[p \overline{D_{2} p}\right]-\partial_{2}\left[p \overline{D_{1} p}\right]\right) .
\end{aligned}
$$

Hence the energy has a lower bound

$$
E=\int_{\mathbb{R}^{2}} \mathcal{E} \mathrm{d} x \geq \pm(a \xi+c \zeta) \int_{\mathbb{R}^{2}} \hat{F}_{12} \mathrm{~d} x \pm(b \xi+d \zeta) \int_{\mathbb{R}^{2}} \tilde{F}_{12} \mathrm{~d} x,
$$

which is attained only if the field configuration $\left(q, p, \hat{A}_{j}, \tilde{A}_{j}\right)$ satisfies the first-order equations

$$
\begin{aligned}
\hat{F}_{12} \pm \kappa\left(a\left[|q|^{2}-\xi\right]+c\left[|p|^{2}-\zeta\right]\right) & =0 \\
\tilde{F}_{12} \pm \lambda\left(b\left[|q|^{2}-\xi\right]+d\left[|p|^{2}-\zeta\right]\right) & =0 \\
D_{1} q \pm \mathrm{i} D_{2} q & =0, \\
D_{1} p \pm \mathrm{i} D_{2} p & =0,
\end{aligned}
$$

which are of the BPS type. The Tong-Wong equations (2.17)-(2.20) are seen to correspond to the limiting case $a=1, b=-1, c=0, d=1$ in (3.10)-(3.13). Note also that, in deriving these equations, we do not require the non-degeneracy condition (2.21). The fulfillment of such a condition is required only when we attempt to establish an existence theory for the vortex solutions of the equations, which will be addressed in section 6 . 


\section{Chern-Simons vortices in presence of magnetic impurities}

Adding a magnetic source term as in $[17,46]$, the Abelian Chern-Simons-Higgs Lagrangian density of $[16,18]$ is modified into

$$
\mathcal{L}=-\frac{1}{4} \kappa \varepsilon^{\mu \nu \alpha} A_{\mu} F_{\nu \alpha}+D_{\mu} \phi \overline{D^{\mu} \phi}-\frac{1}{\kappa^{2}}|\phi|^{2}\left(|\phi|^{2}-\sigma(x)-\zeta\right)^{2} \pm \sigma(x) F_{12},
$$

where $\zeta>0$. The Euler-Lagrange equations of (4.1) are

$$
\begin{aligned}
\frac{1}{2} \kappa \varepsilon^{\mu \nu \alpha} F_{\nu \alpha} & =\mathrm{i}\left(\bar{\phi} D^{\mu} \phi-\phi \overline{D^{\mu} \phi}\right)+J_{\sigma}^{\mu} \\
D_{\mu} D^{\mu} \phi & =-\frac{1}{\kappa^{2}}\left(\left(|\phi|^{2}-\sigma-\zeta\right)^{2}+2|\phi|^{2}\left(|\phi|^{2}-\sigma-\zeta\right)\right) \phi
\end{aligned}
$$

where $J_{\sigma}^{\mu}$ is the $\sigma$-generated current density given as

$$
J_{\sigma}^{0}=0, \quad J_{\sigma}^{i}= \pm \varepsilon^{i j} \partial_{j} \sigma .
$$

For static solutions, the $\mu=0$ component of (4.2) is the Chern-Simons Gauss law constraint,

$$
\kappa F_{12}=2 A_{0}|\phi|^{2} \equiv \rho,
$$

which relates the induced magnetic field $F_{12}$ to the electric charge density $\rho$. Thus, in view of (4.5), we see that the energy density becomes

$$
\begin{aligned}
\mathcal{E} & =-\mathcal{L} \\
& =\kappa A_{0} F_{12}-A_{0}^{2}|\phi|^{2}+\left|D_{j} \phi\right|^{2}+\frac{1}{\kappa^{2}}|\phi|^{2}\left(|\phi|^{2}-\zeta-\sigma\right)^{2} \mp \sigma F_{12} \\
& =\frac{\kappa^{2}}{4|\phi|^{2}} F_{12}^{2}+\left|D_{j} \phi\right|^{2}+\frac{1}{\kappa^{2}}|\phi|^{2}\left(|\phi|^{2}-\zeta-\sigma\right)^{2} \mp \sigma F_{12} \\
& =\left(\frac{\kappa}{2} \frac{F_{12}}{|\phi|} \pm \frac{|\phi|}{\kappa}\left(|\phi|^{2}-\zeta-\sigma\right)\right)^{2} \mp F_{12}\left(|\phi|^{2}-\zeta\right)+\left|D_{j} \phi\right|^{2} .
\end{aligned}
$$

As before, we also have

$$
\left|D_{j} \phi\right|^{2}=\left|D_{1} \phi \pm \mathrm{i} D_{2} \phi\right|^{2} \pm \mathrm{i}\left(\partial_{1}\left[\phi \overline{D_{2} \phi}\right]-\partial_{2}\left[\phi \overline{D_{1} \phi}\right]\right) \pm F_{12}|\phi|^{2} .
$$

Combining (4.6) and (4.7), we have

$$
\begin{aligned}
E & =\int_{\mathbb{R}^{2}} \mathcal{E} \mathrm{d} x \\
& =\int_{\mathbb{R}^{2}}\left(\frac{\kappa}{2} \frac{F_{12}}{|\phi|} \pm \frac{|\phi|}{\kappa}\left(|\phi|^{2}-\zeta-\sigma\right)\right)^{2} \mathrm{~d} x+\int_{\mathbb{R}^{2}}\left|D_{1} \phi \pm \mathrm{i} D_{2} \phi\right|^{2} \mathrm{~d} x \pm \zeta \int_{\mathbb{R}^{2}} F_{12} \mathrm{~d} x \\
& \geq 2 \pi \zeta|N|,
\end{aligned}
$$

where the winding $N$ appears again as in (2.11) and the sign convention $|N|= \pm N$ is followed. The saturation of the energy lower bound (4.8) leads to the Chern-Simons BPS vortex equations

$$
\begin{aligned}
D_{1} \phi \pm \mathrm{i} D_{2} \phi & =0, \\
F_{12} \pm \frac{2}{\kappa^{2}}|\phi|^{2}\left(|\phi|^{2}-\zeta-\sigma(x)\right) & =0
\end{aligned}
$$


which contain the classical source-free self-dual Chern-Simons equations $[16,18]$ for $\sigma=0$, as anticipated.

It can be examined directly that (4.9)-(4.10), coupled with (4.5), imply (4.2)-(4.3).

For an $N$-vortex solution, we have in view of (4.5) and (4.8) the formulas expressing the carried magnetic flux, electric charge, and tension, as follows:

$$
\Phi=\int F_{12} \mathrm{~d} x=2 \pi N, \quad Q=\int \rho \mathrm{d} x=2 \pi \kappa N, \quad E=2 \pi \zeta|N| .
$$

\section{Generalized Chern-Simons-Higgs vortices}

We now extend our studies in the previous two sections to the Abelian Chern-SimonsHiggs situation containing two scalar fields $q$ and $p$ of the doublet charges $(a, b)$ and $(c, d)$, respectively. For this purpose, consider the following Lagrangian density

$$
\mathcal{L}=-\frac{\kappa_{1}}{4} \varepsilon^{\mu \nu \alpha} \hat{A}_{\mu} \hat{F}_{\nu \alpha}-\frac{\kappa_{2}}{4} \varepsilon^{\mu \nu \alpha} \tilde{A}_{\mu} \tilde{F}_{\nu \alpha}+D_{\mu} q \overline{D^{\mu} q}+D_{\mu} p \overline{D^{\mu} p}-V\left(|q|^{2},|p|^{2}\right),
$$

where the potential density $V$ is to be specified later.

The equations of motion of (5.1) read

$$
\begin{aligned}
\frac{\kappa_{1}}{2} \varepsilon^{\mu \nu \alpha} \hat{F}_{\nu \alpha} & =\hat{j}^{\mu} \equiv-\mathrm{i}\left(a\left[q \overline{D^{\mu} q}-\bar{q} D^{\mu} q\right]+c\left[p \overline{D^{\mu} p}-\bar{p} D^{\mu} p\right]\right), \\
\frac{\kappa_{2}}{2} \varepsilon^{\mu \nu \alpha} \tilde{F}_{\nu \alpha} & =\tilde{j}^{\mu} \equiv-\mathrm{i}\left(b\left[q \overline{D^{\mu} q}-\bar{q} D^{\mu} q\right]+d\left[p \overline{D^{\mu} p}-\bar{p} D^{\mu} p\right]\right), \\
D_{\mu} D^{\mu} q & =\frac{\partial V\left(|q|^{2},|p|^{2}\right)}{\partial \bar{q}} \\
D_{\mu} D^{\mu} p & =\frac{\partial V\left(|q|^{2},|p|^{2}\right)}{\partial \bar{p}} .
\end{aligned}
$$

The static energy density is given by

$$
\begin{aligned}
\mathcal{E}= & -\mathcal{L} \\
= & \kappa_{1} \hat{A}_{0} \hat{F}_{12}+\kappa_{2} \tilde{A}_{0} \tilde{F}_{12}-\left(a \hat{A}_{0}+b \tilde{A}_{0}\right)^{2}|q|^{2}-\left(c \hat{A}_{0}+d \tilde{A}_{0}\right)^{2}|p|^{2} \\
& +\left|D_{j} q\right|^{2}+\left|D_{j} p\right|^{2}+V\left(|q|^{2},|p|^{2}\right) .
\end{aligned}
$$

The Gauss laws (the $\mu=0$ components of the equations (5.2) and (5.3)) may be read off to be

$$
\begin{aligned}
& \kappa_{1} \hat{F}_{12}=2 a\left(a \hat{A}_{0}+b \tilde{A}_{0}\right)|q|^{2}+2 c\left(c \hat{A}_{0}+d \tilde{A}_{0}\right)|p|^{2}, \\
& \kappa_{2} \tilde{F}_{12}=2 b\left(a \hat{A}_{0}+b \tilde{A}_{0}\right)|q|^{2}+2 d\left(c \hat{A}_{0}+d \tilde{A}_{0}\right)|p|^{2},
\end{aligned}
$$

which can be converted to yield the relations

$$
\begin{aligned}
& \left(a \hat{A}_{0}+b \tilde{A}_{0}\right)|q|^{2}=\frac{1}{2(a d-b c)}\left(d \kappa_{1} \hat{F}_{12}-c \kappa_{2} \tilde{F}_{12}\right), \\
& \left(c \hat{A}_{0}+d \tilde{A}_{0}\right)|p|^{2}=\frac{1}{2(a d-b c)}\left(-b \kappa_{1} \hat{F}_{12}+a \kappa_{2} \tilde{F}_{12}\right),
\end{aligned}
$$


where and in the sequel we observe the non-degeneracy condition (2.21). Consequently, we have

$$
\begin{aligned}
\left(a \hat{A}_{0}\right. & \left.+b \tilde{A}_{0}\right)^{2}|q|^{2}+\left(c \hat{A}_{0}+d \tilde{A}_{0}\right)^{2}|p|^{2} \\
& =\frac{1}{2(a d-b c)}\left\{\left(a \hat{A}_{0}+b \tilde{A}_{0}\right)\left(d \kappa_{1} \hat{F}_{12}-c \kappa_{2} \tilde{F}_{12}\right)+\left(c \hat{A}_{0}+d \tilde{A}_{0}\right)\left(-b \kappa_{1} \hat{F}_{12}+a \kappa_{2} \tilde{F}_{12}\right)\right\} \\
& =\frac{1}{2}\left(\kappa_{1} \hat{A}_{0} \hat{F}_{12}+\kappa_{2} \tilde{A}_{0} \tilde{F}_{12}\right) .
\end{aligned}
$$

Thus the energy density (5.6) may be rewritten as

$$
\begin{aligned}
\mathcal{E}= & \frac{1}{2}\left(\kappa_{1} \hat{A}_{0} \hat{F}_{12}+\kappa_{2} \tilde{A}_{0} \tilde{F}_{12}\right)+\left|D_{j} q\right|^{2}+\left|D_{j} p\right|^{2}+V\left(|q|^{2},|q|^{2}\right) \\
= & \left(a \hat{A}_{0}+b \tilde{A}_{0}\right)^{2}|q|^{2}+\left(c \hat{A}_{0}+d \tilde{A}_{0}\right)^{2}|p|^{2}+\left|D_{j} q\right|^{2}+\left|D_{j} p\right|^{2}+V\left(|q|^{2},|q|^{2}\right) \\
= & \left(\frac{d \kappa_{1} \hat{F}_{12}-c \kappa_{2} \tilde{F}_{12}}{2(a d-b c)|q|}\right)^{2}+\left(\frac{-b \kappa_{1} \hat{F}_{12}+a \kappa_{2} \tilde{F}_{12}}{2(a d-b c)|p|}\right)^{2} \\
& +\left|D_{j} q\right|^{2}+\left|D_{j} p\right|^{2}+V\left(|q|^{2},|p|^{2}\right) .
\end{aligned}
$$

From (5.12) we are led to choosing the potential density

$$
\begin{aligned}
V\left(|q|^{2},|p|^{2}\right)= & |q|^{2}\left(\left[\frac{a^{2}}{\kappa_{1}}+\frac{b^{2}}{\kappa_{2}}\right]\left[|q|^{2}-\xi\right]+\left[\frac{a c}{\kappa_{1}}+\frac{b d}{\kappa_{2}}\right]\left[|p|^{2}-\zeta\right]\right)^{2} \\
& +|p|^{2}\left(\left[\frac{a c}{\kappa_{1}}+\frac{b d}{\kappa_{2}}\right]\left[|q|^{2}-\xi\right]+\left[\frac{c^{2}}{\kappa_{1}}+\frac{d^{2}}{\kappa_{2}}\right]\left[|p|^{2}-\zeta\right]\right)^{2} .
\end{aligned}
$$

Therefore we obtain

$$
\begin{aligned}
\mathcal{E}= & \left(\frac{d \kappa_{1} \hat{F}_{12}-c \kappa_{2} \tilde{F}_{12}}{2(a d-b c)|q|} \pm|q|\left(\left[\frac{a^{2}}{\kappa_{1}}+\frac{b^{2}}{\kappa_{2}}\right]\left[|q|^{2}-\xi\right]+\left[\frac{a c}{\kappa_{1}}+\frac{b d}{\kappa_{2}}\right]\left[|p|^{2}-\zeta\right]\right)\right)^{2} \\
& +\left(\frac{-b \kappa_{1} \hat{F}_{12}+a \kappa_{2} \tilde{F}_{12}}{2(a d-b c)|p|} \pm|p|\left(\left[\frac{a c}{\kappa_{1}}+\frac{b d}{\kappa_{2}}\right]\left[|q|^{2}-\xi\right]+\left[\frac{c^{2}}{\kappa_{1}}+\frac{d^{2}}{\kappa_{2}}\right]\left[|p|^{2}-\zeta\right]\right)\right)^{2} \\
& \mp \frac{1}{a d-b c}\left\{\left(d \kappa_{1} \hat{F}_{12}-c \kappa_{2} \tilde{F}_{12}\right)\left(\left[\frac{a^{2}}{\kappa_{1}}+\frac{b^{2}}{\kappa_{2}}\right]\left[|q|^{2}-\xi\right]+\left[\frac{a c}{\kappa_{1}}+\frac{b d}{\kappa_{2}}\right]\left[|p|^{2}-\zeta\right]\right)\right. \\
& +\left|D_{1} q \pm \mathrm{i} D_{2} q\right|^{2} \pm \mathrm{i}\left(\partial_{1}\left[q \overline{D_{2} q}\right]-\partial_{2}\left[q \overline{\left.D_{1} q\right]}\right) \pm\left(a \hat{F}_{12}+b \tilde{F}_{12}\right)|q|^{2}\right. \\
& +\left|D_{1} p \pm \mathrm{i} D_{2} p\right|^{2} \pm \mathrm{i}\left(\partial_{1}\left[p \overline{D_{2} p}\right]-\partial_{2}\left[p \overline{D_{1} p}\right]\right) \pm\left(c \hat{F}_{12}+d \tilde{F}_{12}\right)|p|^{2} \\
= & \left.\left.\left(\frac{d \kappa_{1} \hat{F}_{12}-c \kappa_{2} \tilde{F}_{12}}{2(a d-b c)|q|} \pm|q|\left(\left[\frac{a^{2}}{\kappa_{1}}+\frac{b^{2}}{\kappa_{2}}\right]\left[|q|^{2}-\xi\right]+\left[\frac{a c}{\kappa_{1}}+\frac{b d}{\kappa_{2}}\right]\left[|p|^{2}-\zeta\right]\right)\right)^{2}\right)\right\} \\
& +\left(\frac{-b \kappa_{1} \hat{F}_{12}+a \kappa_{2} \tilde{F}_{12}}{2(a d-b c)|p|} \pm|p|\left(\left[\frac{a c}{\kappa_{1}}+\frac{b d}{\kappa_{2}}\right]\left[|q|^{2}-\xi\right]+\left[\frac{c^{2}}{\kappa_{1}}+\frac{d^{2}}{\kappa_{2}}\right]\left[|p|^{2}-\zeta\right]\right)\right)^{2} \\
& +\left|D_{1} q \pm \mathrm{i} D_{2} q\right|^{2} \pm \mathrm{i}\left(\partial_{1}\left[q \overline{D_{2} q}\right]-\partial_{2}\left[q \overline{D_{1} q}\right]\right) \pm(a \xi+c \zeta) \hat{F}_{12} \\
& +\left|D_{1} p \pm \mathrm{i} D_{2} p\right|^{2} \pm \mathrm{i}\left(\partial_{1}\left[p \overline{D_{2} p}\right]-\partial_{2}\left[p \overline{D_{1} p}\right]\right) \pm(b \xi+d \zeta) \tilde{F}_{12} .
\end{aligned}
$$


So the energy admits a lower bound

$$
E=\int_{\mathbb{R}^{2}} \mathcal{E} \mathrm{d} x \geq \pm(a \xi+c \zeta) \int_{\mathbb{R}^{2}} \hat{F}_{12} \mathrm{~d} x \pm(b \xi+d \zeta) \int_{\mathbb{R}^{2}} \tilde{F}_{12} \mathrm{~d} x,
$$

which is saturated only if the following first-order equations hold

$$
\begin{aligned}
\frac{d \kappa_{1} \hat{F}_{12}-c \kappa_{2} \tilde{F}_{12}}{2(a d-b c)} \pm|q|^{2}\left(\left[\frac{a^{2}}{\kappa_{1}}+\frac{b^{2}}{\kappa_{2}}\right]\left[|q|^{2}-\xi\right]+\left[\frac{a c}{\kappa_{1}}+\frac{b d}{\kappa_{2}}\right]\left[|p|^{2}-\zeta\right]\right) & =0, \\
\frac{-b \kappa_{1} \hat{F}_{12}+a \kappa_{2} \tilde{F}_{12}}{2(a d-b c)} \pm|p|^{2}\left(\left[\frac{a c}{\kappa_{1}}+\frac{b d}{\kappa_{2}}\right]\left[|q|^{2}-\xi\right]+\left[\frac{c^{2}}{\kappa_{1}}+\frac{d^{2}}{\kappa_{2}}\right]\left[|p|^{2}-\zeta\right]\right) & =0, \\
D_{1} q \pm \mathrm{i} D_{2} q & =0, \\
D_{1} p \pm \mathrm{i} D_{2} p & =0 .
\end{aligned}
$$

We note that the equations (5.16)-(5.17) may be rearranged such that $\hat{F}_{12}$ and $\tilde{F}_{12}$ stand out to be expressed explicitly in terms of $|q|^{2}$ and $|p|^{2}$ :

$$
\begin{aligned}
\hat{F}_{12} & \pm \frac{2 a}{\kappa_{1}}|q|^{2}\left(\left[\frac{a^{2}}{\kappa_{1}}+\frac{b^{2}}{\kappa_{2}}\right]\left[|q|^{2}-\xi\right]+\left[\frac{a c}{\kappa_{1}}+\frac{b d}{\kappa_{2}}\right]\left[|p|^{2}-\zeta\right]\right) \\
& \pm \frac{2 c}{\kappa_{1}}|p|^{2}\left(\left[\frac{a c}{\kappa_{1}}+\frac{b d}{\kappa_{2}}\right]\left[|q|^{2}-\xi\right]+\left[\frac{c^{2}}{\kappa_{1}}+\frac{d^{2}}{\kappa_{2}}\right]\left[|p|^{2}-\zeta\right]\right)=0, \\
\tilde{F}_{12} & \pm \frac{2 b}{\kappa_{2}}|q|^{2}\left(\left[\frac{a^{2}}{\kappa_{1}}+\frac{b^{2}}{\kappa_{2}}\right]\left[|q|^{2}-\xi\right]+\left[\frac{a c}{\kappa_{1}}+\frac{b d}{\kappa_{2}}\right]\left[|p|^{2}-\zeta\right]\right) \\
& \pm \frac{2 d}{\kappa_{2}}|p|^{2}\left(\left[\frac{a c}{\kappa_{1}}+\frac{b d}{\kappa_{2}}\right]\left[|q|^{2}-\xi\right]+\left[\frac{c^{2}}{\kappa_{1}}+\frac{d^{2}}{\kappa_{2}}\right]\left[|p|^{2}-\zeta\right]\right)=0 .
\end{aligned}
$$

Alternatively these two equations may be rewritten usefully as

$$
\begin{aligned}
a \hat{F}_{12}+b \tilde{F}_{12} & \pm 2\left(\frac{a^{2}}{\kappa_{1}}+\frac{b^{2}}{\kappa_{2}}\right)|q|^{2}\left(\left[\frac{a^{2}}{\kappa_{1}}+\frac{b^{2}}{\kappa_{2}}\right]\left[|q|^{2}-\xi\right]+\left[\frac{a c}{\kappa_{1}}+\frac{b d}{\kappa_{2}}\right]\left[|p|^{2}-\zeta\right]\right) \\
& \pm 2\left(\frac{a c}{\kappa_{1}}+\frac{b d}{\kappa_{2}}\right)|p|^{2}\left(\left[\frac{a c}{\kappa_{1}}+\frac{b d}{\kappa_{2}}\right]\left[|q|^{2}-\xi\right]+\left[\frac{c^{2}}{\kappa_{1}}+\frac{d^{2}}{\kappa_{2}}\right]\left[|p|^{2}-\zeta\right]\right)=0 \\
c \hat{F}_{12}+d \tilde{F}_{12} & \pm 2\left(\frac{a c}{\kappa_{1}}+\frac{b d}{\kappa_{2}}\right)|q|^{2}\left(\left[\frac{a^{2}}{\kappa_{1}}+\frac{b^{2}}{\kappa_{2}}\right]\left[|q|^{2}-\xi\right]+\left[\frac{a c}{\kappa_{1}}+\frac{b d}{\kappa_{2}}\right]\left[|p|^{2}-\zeta\right]\right) \\
& \pm 2\left(\frac{b^{2}}{\kappa_{1}}+\frac{d^{2}}{\kappa_{2}}\right)|p|^{2}\left(\left[\frac{a c}{\kappa_{1}}+\frac{b d}{\kappa_{2}}\right]\left[|q|^{2}-\xi\right]+\left[\frac{c^{2}}{\kappa_{1}}+\frac{d^{2}}{\kappa_{2}}\right]\left[|p|^{2}-\zeta\right]\right)=0 .
\end{aligned}
$$

\section{Solutions of vortex equations}

In this section we present a series of rigorous existence results for the BPS vortex equations discussed in the previous sections. In the first subsection, we consider the simplest cases, (2.12)-(2.13) and (4.9)-(4.10). It is seen that, even in such a simple situation, the Abelian Higgs equations and Chern-Simons equations exhibit some sharply different features with respect to the presence of a source term. In the second subsection, we consider the generalized Abelian Higgs equations (3.10)-(3.13). In the last subsection, we consider the generalized Abelian Chern-Simons-Higgs equations (5.16)-(5.19). 


\subsection{Vortex equations in presence of $\sigma(x)$-source terms}

We first consider the system of BPS vortex equations (2.12)-(2.13). From (2.12) we know that the zeros of $\phi$ are finitely many and of algebraic multiplicities. In fact, the total number of zeros, counting multiplicities, is the winding number of $\phi$ near infinity of $\mathbb{R}^{2}$ (cf. [19]). Hence, using

$$
Z_{\phi}=\left\{z_{1}, \ldots, z_{N}\right\}
$$

to denote the set of zeros of $\phi$ (multiple zeros are counted with repetitions), the substitution $u=\ln |\phi|^{2}$ recasts (2.12)-(2.13) into the nonlinear elliptic equation

$$
\Delta u=2 \kappa\left(\mathrm{e}^{u}-\zeta-\sigma(x)\right)+4 \pi \sum_{s=1}^{N} \delta_{z_{s}}(x),
$$

where $\delta_{z}$ denote the usual Dirac measure concentrated at $z$. In [19], an existence and uniqueness theory is developed for this equation when the $\sigma$-term is absent. The presence of the $\sigma$-term in (6.2), on the other hand, introduces some technical complications for obtaining an existence and uniqueness theory under the general finite source-energy condition

$$
\int_{\mathbb{R}^{2}} \sigma^{2}(x) \mathrm{d} x<\infty .
$$

Here, for simplicity, we consider the equation over a doubly-periodic lattice domain, $\Omega$, which may be viewed as a flat 2 -torus. That is, $\Omega$ represents a lattice cell hosting periodically distributed Abrikosov vortices [1], where periodicity is realized by the 't Hooft boundary condition $[42,47]$.

Let $u_{0}$ be a doubly-periodic function over $\Omega$ satisfying [2]

$$
\Delta u_{0}=-\frac{4 \pi N}{|\Omega|}+4 \pi \sum_{s=1}^{N} \delta_{z_{s}}(x),
$$

where $|\Omega|$ denotes the total area of $\Omega$. Then $u=u_{0}+v$ transforms (6.2) over $\Omega$ into the following source-free equation

$$
\Delta v=2 \kappa\left(\mathrm{e}^{u_{0}+v}-\zeta-\sigma(x)\right)+\frac{4 \pi N}{|\Omega|}, \quad x \in \Omega .
$$

Integrating (6.5), we arrive at the natural constraint

$$
\zeta|\Omega|+\int_{\Omega} \sigma(x) \mathrm{d} x-\frac{2 \pi N}{\kappa}=\int_{\Omega} \mathrm{e}^{u_{0}+v} \mathrm{~d} x,
$$

which leads to the necessary condition

$$
\frac{2 \pi N}{\kappa}<\zeta|\Omega|+\int_{\Omega} \sigma(x) \mathrm{d} x .
$$

When $\sigma=0,(6.7)$ is known as the Bradlow bound [5, 25, 28]. See also [30, 31]. Using the methods in $[47,49]$, it is not hard to show that (6.7) is also sufficient to ensure the existence 
of a solution, and that, the solution must be unique. In particular, if we decompose $\sigma$ into the sum of its positive and negative parts, $\sigma_{+}=\max \{\sigma, 0\}$ and $\sigma_{-}=\max \{-\sigma, 0\}$, so that

$$
\sigma=\sigma_{+}-\sigma_{-},
$$

then we see from the bound (6.7) that the number of vortices allowed is enhanced by $\sigma_{+}$ but diminished by $\sigma_{-}$, which is an interesting phenomenon.

Note that another more transparent way, perhaps, to understand (6.7) is to rewrite it as

$$
\frac{2 \pi N}{\kappa|\Omega|}<\zeta+\sigma_{0}
$$

where $\sigma_{0}$ is the average value of $\sigma(x)$ over $\Omega$ :

$$
\sigma_{0}=\frac{1}{|\Omega|} \int_{\Omega} \sigma(x) \mathrm{d} x
$$

Thus (6.9) spells out a necessary and sufficient condition for the average value of the source term $\sigma(x)$ in order that an $N$-vortex solution exists over $\Omega$. In particular, if $\sigma_{0} \leq-\zeta$, no $N$-vortex solution exists for any $\kappa>0$ and domain $\Omega$.

The existence and uniqueness of an $N$-vortex solution realizing $N$ arbitrarily prescribed vortex points given as the zeros of the Higgs scalar $\phi$ indicates that the set of $N$-vortex solutions of the equations (2.12)-(2.13) depends exactly on $2 N$ parameters labeling those zeros.

We next consider the Chern-Simons-Higgs vortex equations (4.9)-(4.10).

Assuming the zero set of $\phi$ is as given in (6.1) and taking $u=\ln |\phi|^{2}$ as before, we reduce (4.9)-(4.10) into

$$
\Delta u=\frac{4}{\kappa^{2}} \mathrm{e}^{u}\left(\mathrm{e}^{u}-\zeta-\sigma(x)\right)+4 \pi \sum_{s=1}^{N} \delta_{z_{s}}(x) .
$$

If the problem is considered over a doubly-periodic domain, $\Omega$, then $u=u_{0}+v$ (where the background function $u_{0}$ satisfies (6.4)) renders (6.11) into

$$
\Delta v=\frac{4}{\kappa^{2}} \mathrm{e}^{u_{0}+v}\left(\mathrm{e}^{u_{0}+v}-\zeta-\sigma(x)\right)+\frac{4 \pi N}{|\Omega|} .
$$

Integrating (6.12), we arrive at the constraint

$$
4 \int_{\Omega}\left(\mathrm{e}^{u_{0}+v}-\frac{(\zeta+\sigma)}{2}\right)^{2} \mathrm{~d} x=\int_{\Omega}(\zeta+\sigma(x))^{2} \mathrm{~d} x-4 \pi N \kappa^{2}
$$

similar to that in the Abelian Higgs model case, which leads us to the necessary condition

$$
\kappa^{2}<\frac{1}{4 \pi N} \int_{\Omega}(\zeta+\sigma(x))^{2} \mathrm{~d} x \equiv \eta_{0}^{2} .
$$

Applying the methods in [6, 49], it can be shown that there is a critical value $0<\kappa_{c}<\eta_{0}$ such that an $N$-vortex solution exists for any $\kappa \in\left(0, \kappa_{c}\right)$ but no solution exists for $\kappa>\kappa_{c}$. 
However, when there is a solution, there is also a secondary solution [43]. Thus the set of solutions of the Chern-Simons-Higgs $N$-vortex equations (4.9)-(4.10) depends on at least $4 N$ parameters.

It is interesting to note that, unlike in the Abelian Higgs situation, $N$-vortices exist when $\kappa$ is small enough as far as

$$
\sigma(x) \not \equiv-\zeta
$$

To end this subsection, we remark that a solution to (6.11) over the full plane $\mathbb{R}^{2}$, with any $\kappa>0$ and $N \geq 1$, satisfying $u \rightarrow \ln \zeta$ as $|x| \rightarrow \infty$ always exists under the finite source-energy condition (6.3).

\subsection{Abelian Higgs vortex equations}

We now consider the BPS equations (3.10)-(3.13). The zero sets of the charged scalar fields $q$ and $p$ are denoted by

$$
Z_{q}=\left\{z_{1}, \ldots, z_{N_{1}}\right\} \quad \text { and } \quad Z_{p}=\left\{\tilde{z}_{1}, \ldots, \tilde{z}_{N_{2}}\right\}
$$

respectively. From the equations (3.12)-(3.13), we have

$$
a \hat{F}_{12}+b \tilde{F}_{12}=\frac{1}{2} \Delta \ln |q|^{2}, \quad c \hat{F}_{12}+d \tilde{F}_{12}=\frac{1}{2} \Delta \ln |p|^{2},
$$

away from the zeros of $q$ and $p$. Let $u=\ln |q|^{2}$ and $v=\ln |p|^{2}$. Then from (3.10)-(3.11) we obtain the following coupled nonlinear elliptic equations

$$
\begin{aligned}
& \Delta u=2\left(\kappa a^{2}+\lambda b^{2}\right)\left(\mathrm{e}^{u}-\xi\right)+2(\kappa a c+\lambda b d)\left(\mathrm{e}^{v}-\zeta\right)+4 \pi \sum_{s=1}^{N_{1}} \delta_{z_{s}}, \\
& \Delta v=2(\kappa a c+\lambda b d)\left(\mathrm{e}^{u}-\xi\right)+2\left(\kappa c^{2}+\lambda d^{2}\right)\left(\mathrm{e}^{v}-\zeta\right)+4 \pi \sum_{s=1}^{N_{2}} \delta_{\tilde{z}_{s}} .
\end{aligned}
$$

Let $K$ be a matrix of the form

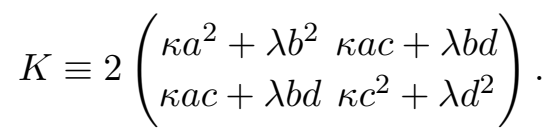

Then we write the equations (6.18)-(6.19) into a compact form

$$
\Delta\left(\begin{array}{l}
u \\
v
\end{array}\right)=K\left(\begin{array}{l}
\mathrm{e}^{u}-\xi \\
\mathrm{e}^{v}-\zeta
\end{array}\right)+4 \pi\left(\begin{array}{l}
\sum_{s=1}^{N_{1}} \delta_{z_{s}} \\
\sum_{s=1}^{N_{2}} \delta_{\tilde{z}_{s}}
\end{array}\right)
$$

Since $\kappa, \lambda>0, a d-b c \neq 0$, the matrix $K$ defined by (6.20) is positive definite. Then we may use the a direct minimization approach developed in [22] to establish the existence and uniqueness results for the equations (6.18)-(6.19) in both planar case and doubly periodic case. These results are summarized as follows. 
(i) On $\mathbb{R}^{2}$ there is a unique solution satisfying the boundary condition $u=\ln \xi, v=\ln \zeta$ at infinity. Moreover, this solution enjoys the exponential decay estimate

$$
(u(x)-\ln \xi)^{2}+(v(x)-\ln \zeta)^{2}+|\nabla u(x)|^{2}+|\nabla v(x)|^{2}=\mathrm{O}\left(\mathrm{e}^{-\sqrt{\lambda_{0} \min \{\xi, \zeta\}}|x|}\right),
$$

as $|x| \rightarrow \infty$, where $\lambda_{0}$ is the smaller eigenvalue of the matrix $K$ with $K$ defined in (6.20).

(ii) On a doubly periodic domain $\Omega$, there exists a solution if and only if

$$
\begin{aligned}
& \frac{\left(\kappa c^{2}+\lambda d^{2}\right) N_{1}-(\kappa a c+\lambda b d) N_{2}}{\kappa \lambda(a d-b c)^{2}}<\frac{\xi|\Omega|}{2 \pi}, \\
& \frac{\left(\kappa a^{2}+\lambda b^{2}\right) N_{2}-(\kappa a c+\lambda b d) N_{1}}{\kappa \lambda(a d-b c)^{2}}<\frac{\zeta|\Omega|}{2 \pi}
\end{aligned}
$$

hold simultaneously. Besides, the solution is unique if it exits.

(iii) In both cases, there hold the quantized integrals

$$
\begin{gathered}
\int\left\{\left(\kappa a^{2}+\lambda b^{2}\right)\left(\mathrm{e}^{u}-\xi\right)+(\kappa a c+\lambda b d)\left(\mathrm{e}^{v}-\zeta\right)\right\} \mathrm{d} x=-2 \pi N_{1}, \\
\int\left\{(\kappa a c+\lambda b d)\left(\mathrm{e}^{u}-\xi\right)+\left(\kappa c^{2}+\lambda d^{2}\right)\left(\mathrm{e}^{v}-\zeta\right)\right\} \mathrm{d} x=-2 \pi N_{2},
\end{gathered}
$$

where the integration is carried out over $\mathbb{R}^{2}$ or $\Omega$.

In view of $(3.10),(3.11),(6.25)$, and (6.26), we obtain the fluxes

$$
\begin{aligned}
& \hat{\Phi}=\int \hat{F}_{12} \mathrm{~d} x=\frac{2 \pi}{(a d-b c)}\left(d N_{1}-b N_{2}\right) \\
& \tilde{\Phi}=\int \tilde{F}_{12} \mathrm{~d} x=\frac{2 \pi}{(a d-b c)}\left(a N_{2}-c N_{1}\right)
\end{aligned}
$$

Thus, using (6.27) and (6.28), we arrive at the exact value of the vortex tension

$$
E=\int \mathcal{E} \mathrm{d} x=2 \pi\left(\xi N_{1}+\zeta N_{2}\right)
$$

It may be checked that (6.23) and (6.24) may be recast into the bounds

$$
\begin{aligned}
& N_{1}<\frac{|\Omega|}{2 \pi}\left(\xi\left[\kappa a^{2}+\lambda b^{2}\right]+\zeta[\kappa a c+\lambda b d]\right), \\
& N_{2}<\frac{|\Omega|}{2 \pi}\left(\xi[\kappa a c+\lambda b d]+\zeta\left[\kappa c^{2}+\lambda d^{2}\right]\right),
\end{aligned}
$$

for the vortex numbers, separately and equivalently, provided that $\kappa a c+\lambda b d>0$ (say). 


\subsection{Chern-Simons vortex equations}

Consider (5.16)-(5.19) so that the sets of zeros of $q$ and $p$ are as prescribed in (6.16). As before the equations (5.18)-(5.19) yield

$$
a \hat{F}_{12}+b \tilde{F}_{12}=\frac{1}{2} \Delta \ln |q|^{2}, \quad c \hat{F}_{12}+d \tilde{F}_{12}=\frac{1}{2} \Delta \ln |p|^{2},
$$

away from the zeros of $q$ and $p$. Set $u=\ln |q|^{2}$ and $v=\ln |p|^{2}$. Hence by (5.22)-(5.23) we obtain the following nonlinear elliptic equations

$$
\begin{aligned}
\Delta u= & 4\left(\frac{a^{2}}{\kappa_{1}}+\frac{b^{2}}{\kappa_{2}}\right) \mathrm{e}^{u}\left(\left[\frac{a^{2}}{\kappa_{1}}+\frac{b^{2}}{\kappa_{2}}\right]\left[\mathrm{e}^{u}-\xi\right]+\left[\frac{a c}{\kappa_{1}}+\frac{b d}{\kappa_{2}}\right]\left[\mathrm{e}^{v}-\zeta\right]\right) \\
& +4\left(\frac{a c}{\kappa_{1}}+\frac{b d}{\kappa_{2}}\right) \mathrm{e}^{v}\left(\left[\frac{a c}{\kappa_{1}}+\frac{b d}{\kappa_{2}}\right]\left[\mathrm{e}^{u}-\xi\right]+\left[\frac{c^{2}}{\kappa_{1}}+\frac{d^{2}}{\kappa_{2}}\right]\left[\mathrm{e}^{v}-\zeta\right]\right) \\
& +4 \pi \sum_{s=1}^{N_{1}} \delta_{z_{s}}, \\
\Delta v= & 4\left(\frac{a c}{\kappa_{1}}+\frac{b d}{\kappa_{2}}\right) \mathrm{e}^{u}\left(\left[\frac{a^{2}}{\kappa_{1}}+\frac{b^{2}}{\kappa_{2}}\right]\left[\mathrm{e}^{u}-\xi\right]+\left[\frac{a c}{\kappa_{1}}+\frac{b d}{\kappa_{2}}\right]\left[\mathrm{e}^{v}-\zeta\right]\right) \\
& +4\left(\frac{b^{2}}{\kappa_{1}}+\frac{d^{2}}{\kappa_{2}}\right) \mathrm{e}^{v}\left(\left[\frac{a c}{\kappa_{1}}+\frac{b d}{\kappa_{2}}\right]\left[\mathrm{e}^{u}-\xi\right]+\left[\frac{c^{2}}{\kappa_{1}}+\frac{d^{2}}{\kappa_{2}}\right]\left[\mathrm{e}^{v}-\zeta\right]\right) \\
& +4 \pi \sum_{s=1}^{N_{2}} \delta_{\tilde{z}_{s}} .
\end{aligned}
$$

These equations can again be rewritten into a vector form

$$
\Delta\left(\begin{array}{l}
u \\
v
\end{array}\right)=K\left(\begin{array}{cc}
\mathrm{e}^{u} & 0 \\
0 & \mathrm{e}^{v}
\end{array}\right) K\left(\begin{array}{l}
\mathrm{e}^{u}-\xi \\
\mathrm{e}^{v}-\zeta
\end{array}\right)+4 \pi\left(\begin{array}{l}
\sum_{s=1}^{N_{1}} \delta_{z_{s}} \\
\sum_{s=1}^{N_{2}} \delta_{\tilde{z}_{s}}
\end{array}\right),
$$

where the matrix $K$ is defined as

$$
K \equiv 2\left(\begin{array}{ll}
\frac{a^{2}}{\kappa_{1}}+\frac{b^{2}}{\kappa_{2}} & \frac{a c}{\kappa_{1}}+\frac{b d}{\kappa_{2}} \\
\frac{a c}{\kappa_{1}}+\frac{b d}{\kappa_{2}} & \frac{c^{2}}{\kappa_{1}}+\frac{d^{2}}{\kappa_{2}}
\end{array}\right) .
$$

Following the approach of [48], we may get an existence theorem for the equations (6.33)(6.34) over $\mathbb{R}^{2}$. The results are summarized as follows.

There exists a solution to the equations (6.33)-(6.34) satisfying the boundary condition $u=\ln \xi, v=\ln \zeta$ at infinity. Moreover, there holds the asymptotic estimate

$$
(u(x)-\ln \xi)^{2}+(v(x)-\ln \zeta)^{2}+|\nabla u(x)|^{2}+|\nabla v(x)|^{2}=\mathrm{O}\left(\mathrm{e}^{-\sqrt{\lambda_{0} \min \{\xi, \zeta\}}|x|}\right),
$$

near infinity, where $\lambda_{0}$ is the smaller eigenvalue of the matrix $K \operatorname{diag}(\xi, \zeta) K$ with $K$ being given by (6.36). Furthermore, for the solution obtained, there hold the quantized integrals

$$
\int K\left(\begin{array}{cc}
\mathrm{e}^{u} & 0 \\
0 & \mathrm{e}^{v}
\end{array}\right) K\left(\begin{array}{c}
\mathrm{e}^{u}-\xi \\
\mathrm{e}^{v}-\zeta
\end{array}\right) \mathrm{d} x=-4 \pi\left(\begin{array}{c}
N_{1} \\
N_{2}
\end{array}\right),
$$

over $\mathbb{R}^{2}$. 
The existence problem over a doubly-periodic domain $\Omega$ becomes more sophisticated in the general setting. For simplicity, we consider the case where $\kappa_{1}=\kappa_{2}=\kappa$. Then, with

$$
K_{0} \equiv 2\left(\begin{array}{cc}
a^{2}+b^{2} & a c+b d \\
a c+b d & c^{2}+d^{2}
\end{array}\right),
$$

the equations (6.33)-(6.34) take the form

$$
\Delta\left(\begin{array}{l}
u \\
v
\end{array}\right)=\frac{1}{\kappa^{2}} K_{0}\left(\begin{array}{cc}
\mathrm{e}^{u} & 0 \\
0 & \mathrm{e}^{v}
\end{array}\right) K_{0}\left(\begin{array}{l}
\mathrm{e}^{u}-\xi \\
\mathrm{e}^{v}-\zeta
\end{array}\right)+4 \pi\left(\begin{array}{l}
\sum_{s=1}^{N_{1}} \delta_{z_{s}} \\
\sum_{s=1}^{N_{2}} \delta_{\tilde{z}_{s}}
\end{array}\right) .
$$

Using a recently developed approach in [13] we obtain the following existence results, assuming $a c+b d<0$ in addition, for technical reasons.

If the system (6.40) admits a solution over $\Omega$, then there must hold

$$
\kappa^{2}(1,1) K_{0}^{-1}\left(N_{1}, N_{2}\right)^{\tau}<\frac{|\Omega|(\xi, \zeta) K_{0}(\xi, \zeta)^{\tau}}{4 \pi} .
$$

In particular there will be no solution whatsoever when $\kappa$ is not sufficiently small. On the other hand, there exists a suitably small positive constant $\kappa_{0}$ such that, when $0<\kappa<\kappa_{0}$, the equation (6.40) has a solution. Moreover, the quantized integrals (6.38) are valid for any $\kappa_{1}, \kappa_{2}>0$ over $\Omega$ as well.

Using (6.38) and (5.20)-(5.21), we immediately obtain the same flux formulas (6.27) and (6.28) as in the extended Abelian Higgs vortex equation situation. Similarly, we see that the same vortex tension formula (6.29) holds as well for the Abelian Chern-Simons vortex equations here.

\section{Conclusions}

In this paper we investigated several extended directions inspired by the recent studies on the Abelian Higgs vortices in the presence of a magnetic source term $[17,46]$ and coupled with an additional Abelian gauge field sector [46].

(i) We showed that the Abelian Higgs equations with a $\sigma$-term $[17,46]$ may be extended into the Abelian Chern-Simons-Higgs formalism [16, 18]. In the former case, for vortices over a periodic lattice cell, there is a specific range for the average value of $\sigma$ which allows vortices to exist and the set of $N$-vortex solutions depends on exactly $2 N$ continuous parameters. In the latter case, however, except in the critical situation when $\sigma=-\zeta$ where $|\phi|^{2}=\zeta$ is the symmetry-breaking ground state, vortices always exist under suitable conditions among coupling constants, vortex number, and domain area, and the set of $N$-vortex solutions depends on at least $4 N$ parameters.

(ii) We showed that the product Abelian Higgs model of Tong and Wong [46], proposed to realize magnetic impurities as heavy, frozen vortices residing in a secondary gauge 
group, described by two Higgs scalar fields, $q$ and $p$, carrying charges $(+1,-1)$ and $(0,+1)$, respectively, may be extended into a general model in which $q$ and $p$ carry charges $(a, b)$ and $(c, d)$ for any $a, b, c, d \in \mathbb{R}$, respectively, and that this general model also possesses a system of BPS equations as a significant reduction from the original equations of motion.

(iii) We also showed that the formalism of Tong and Wong [46] may be extended to the context of the self-dual or BPS Abelian Chern-Simons-Higgs theory, pioneered in the earlier works by Hong, Kim, and Pac [16], and Jackiw and Weinberg [18]. Using these ideas, we developed a product Abelian Chern-Simons-Higgs model hosting two scalar fields $q$ and $p$ carrying charges $(a, b)$ and $(c, d)$, respectively, subject to the non-degeneracy condition $a d-b c \neq 0$.

(iv) For both the extended Abelian Higgs and Chern-Simons-Higgs BPS vortex equations we have established existence results for solutions under the non-degeneracy condition, $a d-b c \neq 0$, and calculated the exact values of the fluxes and energy or tension of a multiple vortex solution, either over a doubly-periodic domain or the full plane.

\section{Acknowledgments}

Han was partially supported by National Natural Science Foundation of China under Grant 11201118 and the Key Foundation for Henan colleges under Grant 15A110013. Both authors were partially supported by National Natural Science Foundation of China under Grants 11471100 and 11471099.

Open Access. This article is distributed under the terms of the Creative Commons Attribution License (CC-BY 4.0), which permits any use, distribution and reproduction in any medium, provided the original author(s) and source are credited.

\section{References}

[1] A.A. Abrikosov, On the magnetic properties of superconductors of the second group, Sov. Phys. JETP 5 (1957) 1174 [INSPIRE].

[2] T. Aubin, Nonlinear analysis on manifolds: Monge-Ampère equations, Springer, Berlin and New York (1982).

[3] R. Auzzi, S. Bolognesi, J. Evslin, K. Konishi and A. Yung, Nonabelian superconductors: vortices and confinement in $\mathcal{N}=2$ SQCD, Nucl. Phys. B 673 (2003) 187 [hep-th/0307287] [INSPIRE].

[4] E.B. Bogomol'nyi, Stability of classical solutions, Sov. J. Nucl. Phys. 24 (1976) 449 [INSPIRE].

[5] S.B. Bradlow, Vortices in holomorphic line bundles over closed Kähler manifolds, Commun. Math. Phys. 135 (1990) 1 [INSPIRE].

[6] L.A. Caffarelli and Y. Yang, Vortex condensation in the Chern-Simons Higgs model: an existence theorem, Commun. Math. Phys. 168 (1995) 321 [InSPIRE]. 
[7] M. Eto, T. Fujimori, S.B. Gudnason, K. Konishi, M. Nitta, K. Ohashi and W. Vinci, Constructing non-Abelian vortices with arbitrary gauge groups, Phys. Lett. B 669 (2008) 98 [arXiv:0802.1020] [INSPIRE].

[8] M. Eto, T. Fujimori, T. Nagashima, M. Nitta, K. Ohashi and N. Sakai, Multiple layer structure of non-Abelian vortex, Phys. Lett. B 678 (2009) 254 [arXiv:0903.1518] [INSPIRE].

[9] M. Eto, Y. Isozumi, M. Nitta, K. Ohashi and N. Sakai, Solitons in the Higgs phase: the moduli matrix approach, J. Phys. A 39 (2006) R315 [hep-th/0602170] [INSPIRE].

[10] M. Eto, Y. Isozumi, M. Nitta, K. Ohashi and N. Sakai, Moduli space of non-Abelian vortices, Phys. Rev. Lett. 96 (2006) 161601 [hep-th/0511088] [INSPIRE].

[11] J. Greensite, An introduction to the confinement problem, Lecture Notes in Physics, volume 821, Springer-Verlag, Berlin and New York (2011).

[12] S.B. Gudnason, Y. Jiang and K. Konishi, Non-Abelian vortex dynamics: effective world-sheet action, JHEP 08 (2010) 012 [arXiv: 1007.2116] [INSPIRE].

[13] X. Han, C.-S. Lin and Y. Yang, Resolution of Chern-Simons-Higgs vortex equations, to appear on Commun. Math. Phys. [arXiv:1503.08330] [InSPIRE].

[14] A. Hanany and D. Tong, Vortices, instantons and branes, JHEP 07 (2003) 037 [hep-th/0306150] [INSPIRE].

[15] A. Hanany and D. Tong, Vortex strings and four-dimensional gauge dynamics, JHEP 04 (2004) 066 [hep-th/0403158] [INSPIRE].

[16] J. Hong, Y. Kim and P.-Y. Pac, Multivortex solutions of the Abelian Chern-Simons-Higgs theory, Phys. Rev. Lett. 64 (1990) 2330.

[17] A. Hook, S. Kachru and G. Torroba, Supersymmetric defect models and mirror symmetry, JHEP 11 (2013) 004 [arXiv:1308.4416] [INSPIRE].

[18] J.G. Amar and F. Family, Amar and Family reply, Phys. Rev. Lett. 64 (1990) 2334 [INSPIRE].

[19] A. Jaffe and C.H. Taubes, Vortices and monopoles, Birkhäuser, Boston U.S.A. (1980).

[20] B. Julia and A. Zee, Poles with both magnetic and electric charges in non-Abelian gauge theory, Phys. Rev. D 11 (1975) 2227 [INSPIRE].

[21] K. Konishi, Advent of non-Abelian vortices and monopoles: further thoughts about duality and confinement, Prog. Theor. Phys. Suppl. 177 (2009) 83 [arXiv:0809.1370] [InSPIRE].

[22] E.H. Lieb and Y. Yang, Non-Abelian vortices in supersymmetric gauge field theory via direct methods, Commun. Math. Phys. 313 (2012) 445 [arXiv:1106.1626] [InSPIRE].

[23] S. Mandelstam, Vortices and quark confinement in non-Abelian gauge theories, Phys. Lett. B 53 (1975) 476 [INSPIRE].

[24] S. Mandelstam, General introduction to confinement, Phys. Rept. C 67 (1980) 109.

[25] N.S. Manton and N.A. Rink, Geometry and energy of non-Abelian vortices, J. Math. Phys. 52 (2011) 043511 [arXiv: 1012.3014] [InSPIRE].

[26] A. Marshakov and A. Yung, Non-Abelian confinement via Abelian flux tubes in softly broken $\mathcal{N}=2$ SUSY QCD, Nucl. Phys. B 647 (2002) 3 [hep-th/0202172] [INSPIRE].

[27] Y. Nambu, Strings, monopoles and gauge fields, Phys. Rev. D 10 (1974) 4262 [INSPIRE].

[28] S.M. Nasir, Vortices and flat connections, Phys. Lett. B 419 (1998) 253 [hep-th/9807020] [INSPIRE]. 
[29] H.B. Nielsen and P. Olesen, Vortex line models for dual strings, Nucl. Phys. B 61 (1973) 45 [INSPIRE].

[30] M. Noguchi, Abelian Higgs theory on Riemann surfaces, Thesis, Duke University (1985).

[31] M. Noguchi, Yang-Mills Higgs theory on a compact Riemann surface, J. Math. Phys. 28 (1987) 2343 [INSPIRE].

[32] M.K. Prasad and C.M. Sommerfield, An exact classical solution for the 't Hooft monopole and the Julia-Zee dyon, Phys. Rev. Lett. 35 (1975) 760 [InSPIRE].

[33] N. Seiberg and E. Witten, Electric-magnetic duality, monopole condensation and confinement in $N=2$ supersymmetric Yang-Mills theory, Nucl. Phys. B 426 (1994) 19 [Erratum ibid. B 430 (1994) 485] [hep-th/9407087] [INSPIRE].

[34] M. Shifman and M. Ünsal, Confinement in Yang-Mills: elements of a big picture, Nucl. Phys. Proc. Suppl. 186 (2009) 235 [arXiv:0810.3861] [InSPIRE].

[35] M. Shifman and A. Yung, Non-Abelian string junctions as confined monopoles, Phys. Rev. D 70 (2004) 045004 [hep-th/0403149] [InSPIRE].

[36] M. Shifman and A. Yung, Localization of non-Abelian gauge fields on domain walls at weak coupling: D-brane prototypes, Phys. Rev. D 70 (2004) 025013 [hep-th/0312257] [INSPIRE].

[37] M. Shifman and A. Yung, Supersymmetric solitons and how they help us understand non-Abelian gauge theories, Rev. Mod. Phys. 79 (2007) 1139 [hep-th/0703267] [INSPIRE].

[38] M. Shifman and A. Yung, Supersymmetric solitons, Cambridge University Press, Cambridge U.K. (2009).

[39] J. Spruck and Y. Yang, Proof of the Julia-Zee theorem, Commun. Math. Phys. 291 (2009) 347 [arXiv:0810.1076] [InSPIRE].

[40] G. 't Hooft, Magnetic monopoles in unified gauge theories, Nucl. Phys. B 79 (1974) 276 [INSPIRE].

[41] G. 't Hooft, On the phase transition towards permanent quark confinement, Nucl. Phys. B 138 (1978) 1 [inSPIRE].

[42] G. 't Hooft, A property of electric and magnetic flux in non-Abelian gauge theories, Nucl. Phys. B 153 (1979) 141 [inSPIRE].

[43] G. Tarantello, Multiple condensate solutions for the Chern-Simons-Higgs theory, J. Math. Phys. 37 (1996) 3769 [INSPIRE].

[44] D. Tong, TASI lectures on solitons: instantons, monopoles, vortices and kinks, hep-th/0509216 [INSPIRE].

[45] D. Tong, Quantum vortex strings: a review, Annals Phys. 324 (2009) 30 [arXiv:0809.5060] [INSPIRE].

[46] D. Tong and K. Wong, Vortices and impurities, JHEP 01 (2014) 090 [arXiv: 1309. 2644] [INSPIRE].

[47] S. Wang and Y. Yang, Abrikosov's vortices in the critical coupling, SIAM J. Math. Anal. 23 (1992) 1125.

[48] Y. Yang, The relativistic non-Abelian Chern-Simons equations, Commun. Math. Phys. 186 (1997) 199 [inSPIRE].

[49] Y. Yang, Solitons in field theory and nonlinear analysis, Springer-Verlag, New York U.S.A. (2001). 